\title{
Two Connections Between Random Systems and Non-Gibbsian Measures
}

\author{
Aernout C. D. van Enter ${ }^{1}$ and Christof Külske ${ }^{2}$
}

Received February 17, 2006; accepted July 18, 2006

Published Online: August 8, 2006

\begin{abstract}
In this contribution we discuss the role disordered (or random) systems have played in the study of non-Gibbsian measures. This role has two main aspects, the distinction between which has not always been fully clear: 1) From disordered systems: Disordered systems can be used as a tool; analogies with, as well as results and methods from the study of random systems can be employed to investigate non-Gibbsian properties of a variety of measures of physical and mathematical interest. 2) $O f$ disordered systems: NonGibbsianness is a property of various (joint) measures describing quenched disordered systems. We discuss and review this distinction and a number of results related to these issues. Moreover, we discuss the mean-field version of the non-Gibbsian property, and present some ideas how a Kac limit approach might connect the finite-range and the mean-field non-Gibbsian properties.
\end{abstract}

KEY WORDS: quenched disorder, non-Gibbsian measures, Kac limits.

\section{INTRODUCTION}

In various situations in physics where one tries to describe many-particle systems, a description of the system as an equilibrium (Gibbs) state in terms of an effective Hamiltonian or an effective temperature is desirable. It has turned out, however, that such a description is not always available. Indeed, one may run into nonGibbsian states, where such a well-behaved Hamiltonian description simply does not exist.

\footnotetext{
${ }^{1}$ Centre for Theoretical Physics, Rijksuniversiteit Groningen, Nijenborgh 4, 9747 AG Groningen, The Netherlands; e-mail: aenter@phys.rug.nl.

${ }^{2}$ Department of Mathematics and Computer Science, Rijksuniversiteit Groningen Blauwborgje 3, 9747 AC Groningen, The Netherlands; e-mail: kuelske@math.rug.nl.
} 
In a classical lattice set-up, non-Gibbsian states — which then are probability measures on (spin-or particle) configuration spaces - have been a subject of considerable interest for the last 15 years or so, see e.g. refs. 15, 16, 22, 28, 30, 42, 43, 46, 51 and also 48 and references therein.

The original study of ref. 15 was mainly motivated by Renormalization Group Transformation existence questions, where the findings are that renormalized measures can be non-Gibbsian. This implies that in these situations a renormalized interaction does not exist. Thus the whole phenomenology of Renormalization Group fixed points, critical exponents expressible in terms of eigenvalues of some Renormalization Group operator in a space of Hamiltonians, its domains of attraction as universality classes, may become problematical once one tries to justify it in a rigorous manner.

Non-Gibbsian measures showed also up in other cases of physical and/or mathematical interest, such as non-equilibrium states in either the transient or the steady-state regime, see e.g. refs. 14, 16, 41, 43, 45. Yet further occurrences are for example in the study of Hidden Markov fields, and in Fortuin-Kasteleyn random-cluster models.

In the mathematical study of these objects, at various occasions results from disordered systems, in particular the phase transition proof of the Random Field Ising Model and possible generalizations thereof ${ }^{(10,52)}$ have been employed. This often is a question of mathematical convenience, and sometimes may be avoidable.

Also, the fact that the non-Gibbsian properties are due to a measure-zero set of "bad" configurations, that is spin-configurations where an presumed effective (renormalized) interaction diverges, shows a certain similarity to the fact, familiar from the study of disordered systems, that various physical properties of interest can be shown to hold only for almost all realizations of the disorder, but not for all of them, and that at the same time this zero-measure set is responsible for some subtle, more singular properties.

This phenomenon is probably best known from the analysis of the Griffiths singularity. ${ }^{(26)}$ This analogy has been explored in e.g. refs. $1-3,11,12$.

A connection of different type was found first in ref. 19 and then further explored in refs. $18,34,36,37,40$. This is the observation that the quenched (joint) measures of disordered systems themselves have non-Gibbsian properties and in fact this non-Gibbsianness can be more severe that what is usually found in the Renormalization Group setting. ${ }^{(40)}$

Some of these considerations have been extended to a mean-field setting where explicit computations can be made. We conjecture that in considerable generality mean-field results correspond well to the non-Gibbsian properties for lattice models in the limit of long-range (Kac-)interactions.

In this paper we review a number of these aspects. 


\section{NOTATION AND BACKGROUND}

For general background on the theory of Gibbs measures we refer to refs. 15,24 . We will mostly consider finite-spin models, living on a finitedimensional lattice $\mathbb{Z}^{d}$. The spins will take the values in a single-spin space $\Omega_{0}$ and we will use small Greek letters $\sigma, \eta, \ldots$ to denote spin configurations for finite or infinite sets of sites. The Hamiltonians in a finite volume $\Lambda \subset \mathbb{Z}^{d}$ with boundary conditions $\eta$ outside will be given by

$$
H^{\Lambda}(\sigma, \eta)=\sum_{X \subset \Lambda} \Phi_{X}\left([\sigma \eta]_{X}\right)
$$

For fixed boundary condition these are functions on the configuration spaces. $\Omega^{\Lambda}=\{-1,1\}^{\Lambda}$. Gibbs measures for an interaction $\Phi$ are probability measures on $\Omega_{0}^{\mathbb{Z}^{d}}$ which have conditional probabilities of finding $\sigma$ inside $\Lambda$, given boundary condition $\eta$ outside $\Lambda$ which have a continuous version, which are of the Gibbsian form

$$
\frac{\exp -H^{\Lambda}(\sigma, \eta)}{Z^{\Lambda}(\eta)}
$$

This should be true for each volume $\Lambda$ and each boundary condition $\eta$. One requires the uniform summability condition on the interaction

$$
\|\Phi\|=\sum_{0 \in X}\left\|\Phi_{X}\right\|_{\infty} \leq \infty
$$

A necessary and (nearly) sufficient condition for a probability measure $P$ to be such a Gibbs measure is that such conditional probabilities are continuous in the product topology - it is then said to to be quasilocal or almost Markovian. It can be shown that being Gibbsian is topologically exceptional in the set of all (or all translation-ergodic) probability measures. ${ }^{(28)}$

To a Gibbs measure can be associated at most one interaction. If a translationinvariant measure has relative entropy density zero (it is always larger or equal than zero) with respect to a given translation-invariant Gibbs measure, it then has the same large deviation properties and it is Gibbs for the same interaction (variational principle).

To show that a particular measure is non-Gibbsian, it is sufficient to find at least one point of discontinuity, that is at least one spin configuration $\omega$, such that

$$
\sup _{\eta^{1}, \eta^{2}}|\mu|\left(\sigma_{0} \mid \omega_{\Lambda} \eta_{\Lambda^{c}}^{1}\right)-\mu\left(\sigma_{0} \mid \omega_{\Lambda} \eta_{\Lambda^{c}}^{2}\right) \mid \geq \epsilon,
$$

uniformly in $\Lambda$. Such an $\omega$ one calls a bad configuration. In many, although not all, examples, these bad configurations have measure zero (almost Gibbs). A weaker property says that one can construct an effective potential which is summable except on a measure zero set (weak Gibbs). ${ }^{(21,40,46)}$ If one considers 
transformed measures on an image-spin space (transformed can mean deterministically or stochastically renormalized or evolved, imperfectly observed ...), one of the typical proofs of non-Gibbsianness is to consider the original measure conditioned on (or constrained by) an image-spin configuration. Once one finds such an image-spin configuration for which a first-order phase transition occurs for the conditioned system, this usually will be a bad configuration, and the existence of such a bad configuration then implies non-Gibbsianness of the transformed measure (see for details in particular, ${ }^{(15)}$ Sec. 4.2).

For disordered models, where next to the spins there are disorder variables, such that the interactions, Hamiltonians, measures, etc. are themselves random objects (they are disorder-dependent), we will denote these disorder variables by $n$ 's. Quenched systems will be described by probability measures on the product space of the disorder and the spin variables. The disorder variables are typically i.i.d. (they describe degrees of freedom which are supposed to be frozen after a quench from - infinitely - high temperature), and the measures describing the quenched systems are defined such that the conditional measures on the spin configurations, given the disorder configuration, are Gibbs measures. Whether or not a configuration is bad now turns out to depend only on the disorder. That is to say, the configuration consists of both spin and disorder variables, but the goodness or badness of a particular configuration only depends on the disorder part and the spin part does not play any role. (This was proved in ref. 34 under the assumption that spin and disorder variables couple in a local way, which holds for all models of interest.)

\section{INPUT FROM RANDOM SYSTEMS FOR NON-GIBBSIANNESS}

\subsection{Finding Bad Configurations; Maps Random and Nonrandom}

In various cases, the finding of a bad configuration for a given measure cannot be done explicitly. However, one can show that a random choice from some Bernoulli measure will do the job. That is, conditioning on a typical random realization of the renormalized or evolved spins can induce a phase transition in the original system, ${ }^{(15,16)}$ once it is conditioned. The existence of this phase transition often follows from refs. 10, 52. Then these typical realizations are all bad.

This strategy especially applies if one wants a result for a continuum of parameters, such as the magnetic field of the untransformed measure, or the bias parameter in an asymmetric evolution. As one can continuously vary the mean of the Bernoulli measure one draws the realization from, one is then left with a continuous family of quenched random models.

As an example, consider a Gibbs measure for the standard nearest neighbor Ising model in dimension $d$ at low temperature. Apply an infinite-temperature 
Glauber dynamics (independent spin flips at Poissonian times) acting on it. Denote the spins at time 0 by $\sigma$ 's and the evolved spins at time $t$ by $\eta$ 's.

Then, fixing the evolved spin at site $i$ at time $t, \eta_{i}(t)$ to be plus or minus, induces an extra bias, that is an extra magnetic field in the plus or minus direction acting on the $\sigma$ spin at site $i$. The strength of these local (dynamical) fields decreases to zero with increasing time. ${ }^{(16)}$ Choosing the $\eta$-configuration to be alternating - a chessboard configuration- means that the study of the conditioned system means considering an Ising system in an alternating field.

When the dynamical field strength $h(t)$ is weak enough — which corresponds to the time being large enough, there is a phase transition in the conditioned system for any dimension at least 2 . Thus the chessboard configuration is in that case a bad configuration for the evolved measure.

If, on the other hand, instead of a chessboard configuration one would choose a random realization from a symmetric Bernoulli measure, we obtain an Ising model in a random, instead of an alternating, magnetic field and one obtains a phase transition for a measure-one set of such realizations, but now only in dimension 3 or more. ${ }^{(10)}$ In this way one obtains an uncountable set of bad configurations.

As a side remark, note that in general any finite-volume perturbation of a bad configuration is again a bad configuration. This also gives rise to an infinite class of bad configurations associated to every bad configuration. This observation, however, only provides a countable set starting from a single configuration.

If the original system was subjected to a weak external magnetic field, one can use a choice from a biased (in the opposite direction) Bernoulli measure, to compensate for this, and again obtain a phase transition. This means considering an Ising model in a non-symmetrically distributed random field, and, as announced in ref. 52, the results of ref. 10 still hold. In this situation one can at the same time vary the external field and the mean of the compensating Bernoulli measure, and one obtains in this way non-Gibbsianness for a continuum of values of the original magnetic fields. This works as long as the dynamical field strength is neither much stronger nor much weaker than the initial field, thus the evolved measure is non-Gibbsian for a finite time interval.

(In physical terminology, when one tries to heat the system fast from cold to hot-the limit measure to which one converges exponentially fast is a Gibbs measure, at infinite, thus very high, temperature, non-Gibbsianness means that at intermediate times one has no temperature, rather than an intermediate temperature.)

The loss of the Gibbs property has also been proved for a diffusive timeevolution, starting from a low-temperature phase, for a model of continuous unbounded spins in a double-well potential. ${ }^{(41)}$ This is a related example, where 
non-Gibbsianness, however, does not rely on random field arguments, and is proved to appear in dimensions $d \geq 2$. Indeed, in this model one can always find a bad conditioning configuration $\eta$ for the time-evolved measure that is homogenous in space. This is possible because of the continuous nature of the spins. To prove non-Gibbsianness it suffices to consider (essentially) translation-invariant models and avoid disordered models.

Another difference with the results for the Ising model is that there is no recovery of the Gibbs-property for large times, in the case of non-zero initial magnetic field. Indeed the bad configurations are diverging with time to infinity, which is only possible for unbounded spins.

Arguments which are mathematically very similar to the case of the Glauber time-evolution apply also for instance when one considers the behaviour of Gibbs measures under a decimation transformation. ${ }^{(15)}$ In this case, the physical conclusion reads that a renormalized interaction does not exist.

We remark that the random Glauber evolution map (which can also be seen as a single-site random renormalization map) and the deterministic decimation map show very much the same kind of behaviour.

\subsection{Analogies: Measure-One Properties and Multiscale Methods}

In various models it can be shown that the set of bad configurations has (non-Gibbsian) measure zero ("almost Gibbs"). Even if this it not true, one may in given models construct an interaction which is defined almost surely ("weak Gibbs"). By abstract arguments almost Gibbs essentially implies weak Gibbs. ${ }^{(46)}$

It has been observed that there is a similarity with phenomenona one knows from disordered systems where the physics is described by the behaviour of the system for a set of measure one from the disorder realizations. One is then interested in the typical (probability one) behaviour of the system.

In fact the similarity goes further, in the sense that certain configurations can locally be good at different scales. E.g. in the Griffiths singularity problem, a disorder configuration configuration consists of occupied clusters, and one finds occupied clusters at arbitrary scales, while in the non-Gibbsian set-up, one can find configurations where the effective interaction reaching the origin has ranges at various scales. This observation has led to similar techniques being applied to both types of problems. In particular the method of multiscale cluster expansions has turned out especially useful in both the study of disordered systems and the study of non-Gibbsian but weak Gibbsian measures. For some examples see e.g. refs. $1-3,11-13,20,23$. 


\section{NON-GIBBSIAN PROPERTIES OF RANDOM SYSTEMS}

\subsection{Quenched Measures and the Morita Approach}

In ref. 19, it was discovered that if one considers the joint measure of a site diluted Ising model, given by the Hamiltonian

$$
-H(n, \sigma)=\sum_{\langle i, j\rangle} n_{i} n_{j} \sigma_{i} \sigma_{j}
$$

where the $n_{i}$ are 0 or 1 with probability $p$ or $1-p$, and the conditional measure on the spins, given the realization of the occupation variables is of the Gibbsian form, this joint (quenched) measure on the product space of the disorder (occupation) variable space and the spin space, which is the limit of

$$
K(n, \sigma)=P(n) \frac{\exp -H(n, \sigma)}{Z(n)}
$$

is itself not a Gibbs measure as defined above. Informally this means that it can not be written as

$$
K(n, \sigma)=\frac{\exp -\bar{H}(n, \sigma)}{Z}
$$

in the thermodynamic limit with a uniform summable Hamiltonian $\bar{H}(n, \sigma)$, (which should be a function of the pair $(n, \sigma)$ of disorder variables $n$ and spinvariables $\sigma$ ).

In the language of disordered systems, the quenched measure cannot be written as an annealed Gibbs measure for a proper potential depending on the joint variables $(n, \sigma)$.

Warning. We use the terms "quenched" and "annealed" in our paper in the original sense, as describing either fast or slowly cooled systems, as is the standard usage in the (mathematical) physics literature on spin systems. Unfortunately, in some probabilistic literature "quenched" is used for almost sure, and "annealed" for averaged properties. To avoid confusion we stress that this is not our convention.

A bad configuration in this model is for example an occupation configuration of two infinite occupied clusters, separated by an infinite empty interval of thickness one (and spin configuration arbitrary). To see where the nonlocality comes from, for simplicity we first consider the $T=0$ case. Then the ground state of the Ising model on these two semi-infinite clusters is fourfold degenerate. Once one connects the two clusters, it is twofold degenerate. Adding an occupied site in this interval can thus lower the entropy by a finite term $\ln 2$ or not, depending on whether the two clusters have another connection (which can be arbitrarily far away) or not. At sufficiently low temperatures a similar reasoning implies nonGibbsianness of the quenched measure, as the extra free energy due to adding a single site can depend nonlocally on the occupation variable far away. Note that 
the goodness or badness of an $(n, \sigma)$-configuration is due to a nonlocal behaviour of the random partition function $Z(n)$, and only depends on the disorder variable $n$, but not on $\sigma$.

Afterwards in ref. 34 criteria for the absence of the Gibbs property for measures on the $(n, \sigma)$ product space of a general class of quenched disordered models $\mu[n]$ depending on disorder variables $n$ were given.

A good example for this is the random field Ising model, given by the Hamiltonian

$$
-H(n, \sigma)=\sum_{\langle i, j\rangle} \sigma_{i} \sigma_{j}+h \sum_{i} n_{i} \sigma_{i}
$$

where the random fields $n_{i}$ are 1 or -1 with probability $\frac{1}{2}$.

Again we look at the large-volume limits of

$$
K(n, \sigma)=P(n) \frac{\exp -H(n, \sigma)}{Z(n)}
$$

for different spin-boundary conditions.

The class of systems allowed in the analysis of ref. 34 includes also the site-diluted Ising model for arbitrary dilutions $p$, (while ref. 19 was restricted to the small- $p$ regime), bond disordered models etc. It was shown in considerable generality that the failure of this Gibbs property in product space occurs whenever there is a discontinuity in the quenched expectation $\mu[n]$ of the spin-observable in the Hamiltonian that is conjugate to the disorder variable $n$. In the case of the random field Ising model, this is just the expectation of the spin $\sigma_{i}$ taken w.r.t. the random-field dependent Gibbs measure $\mu[n]\left(\sigma_{i}\right)$, viewed as a function of the random fields $n$. In 3 dimensions a typical random field configuration allows both a plus and a minus state (as proved by ref. 10), for small enough $h$ and small enough temperature. As changing the field outside some volume $\Lambda$ to either plus or minus picks out one of the two possibilities, here in fact the set of bad configurations (points of essential discontinuity) has full measure, w.r.t. the joint measure. It consists of a full measure set of fields combined with any spin configuration.

In the physics literature, such an annealed description of quenched disordered systems in terms of an effective (so-called "grand") potential had been introduced by Morita long ago, and has been reinvented and studied at various occasions, see e.g. refs. 31, 33, 47 and also 32, 37. As a uniformly convergent grand potential does not exist in many examples, a controlled application of the Morita method is thus quite problematical.

What happens, however, if one gives up any assumptions on the speed of convergence of the potential depending both on spin and disorder variables, and asks only for convergence, possibly arbitrarily slow, on a set of full measure? In ref. 36 the existence of such a potential was shown by soft (martingale) arguments for disordered general models. The proof however exploits the product structure 
of the model, so that this it would not generalize (say) to renormalized measures. To summarize, for quenched models one loses in general any control when one truncates the Hamiltonian, but it least there is a well-defined Hamiltonian to talk about.

To get any bounds on the speed of convergence of the "grand potential", even on a restricted set of realizations of the disorder, is difficult and model-dependent work is needed that can be hard. In the case of the random field Ising model, this can however be done 40 , building on the renormalization group arguments of ref. 10. It shows the decay of the potential like a stretched exponential for almost any random field configuration $n$.

\subsection{Almost Versus Weak Gibbs, Violating the Variational Principle}

We just saw that although the quenched measures of the RFIM are weak Gibbsian with an almost surely rapidly decaying potential, they have a measureone set of bad configurations. (This holds in 3 dimensions, at low temperature, and small random fields.) The possibility of having a measure-one set of bad configurations, can have severe consequences: A particularly surprising fact is that in this situation the variational principle can be violated. ${ }^{(40)}$ This means that the almost surely defined potentials for the plus measure and the minus measure are not the same, even though the relative entropy density between these two translation-invariant measures is zero. In more physical terms, the different phases have different (almost surely defined) grand potentials.

This is a sharp contradiction to classical Gibbs formalism built around the notion of a uniformly summable potential (3). Here the relative entropy density between two measures vanishes if and only if they have the same conditional probabilities (or in other words: equivalent interaction potentials). The random field Ising model thus clearly shows that the proposed class of weak Gibbs measures is too broad for a variational principle to hold. One needs to strive for a smaller class. Partial, but not final, results have been obtained in this direction: On the positive side, ${ }^{(21)}$ showed the validity of the classical variational principle assuming concentration properties on certain "nice" configurations (invoking assumptions which are weaker than almost Gibbsianness-where it follows from ref. 46, in the spirit of, but stronger than, weak Gibbsianness).

\section{MEAN FIELD}

A related analysis has turned out to be possible for mean-field models of Curie-Weiss type. Product states are trivially Gibbs, and non-trivial combinations thereof have a full set of discontinuity points, and thus are non-Gibbs in a strong sense. ${ }^{(17)}$ However, the interesting approach turns out to replace continuity of the conditioning in the product topology by continuity properties of conditional 
probabilities as a function of empirical averages. ${ }^{(27,37,39)}$ One looks at conditional probabilities in finite volumes and then takes the limit, rather than immediately considering the infinite-volume measures. One value of the empirical average for the magnetization now corresponds with a whole collection of spin configurations. The results are often of similar nature as in the short-range situation, but are more complete in the sense that in many cases the whole phase diagram can be treated. The parallel holds, to the extent that the "hidden" phase-transitions that are responsible for the discontinuities that cause non-Gibbsianness on the lattice occur in the same way as for the mean-field counterpart, assuming e.g. large enough lattice dimensions. Remember however that non-Gibbsianness is expressed in different topologies.

Properties like the full-measure set of discontinuities in the quenched measures in the random field Ising model reappear as large-volume asymptotics of finite-volume probabilities of "bad sets". $(37,38)$

Mean-field systems can serve as an illustration but also as a source of heuristics, suggesting new and sometimes unexpected mechanisms of non-Gibbsianness, as in the example of time-evolved measures, ${ }^{(39)}$ where a symmetry-breaking in the set of bad configurations appears. Thus mean-field computations can be a fertile source, motivating further research.

\subsection{Mean Field-via Kac Limits}

Let us discuss the relation between the notion of non-Gibbsianness on the lattice and in mean field in some more detail. A priori it might not be clear that there should be a close connection, since different topologies are involved when looking at the continuity of various conditional probabilities. To draw a link between lattice and mean-field properties (also) on the level of the present discussion of non-Gibbsianness it should be very interesting to investigate Kacmodels. Kac-models, going back to ref. 29, are defined in terms of long-, but finite range interactions of the form $J_{\gamma}(r) \equiv \gamma^{d} J(\gamma r)$. Here $J(x) \geq 0$ is a nice function, rapidly decaying or of bounded support with $\int J(x) d^{d} x=1$. A main example is the ferromagnetic Kac-Ising model of the form

$$
-H_{\gamma}(\sigma)=\frac{1}{2} \sum_{i, j} J_{\gamma}(i-j) \sigma_{i} \sigma_{j}+h \sum_{i} \sigma_{i}
$$

We shall argue that in many cases the Gibbsian/non-Gibbsian properties should be compatible in a nice way via the Kac limit. More precisely, consider a translationinvariant lattice model with long-range (Kac-) potential, depending on a number of parameters describing the interaction, such as e.g. temperature and field for an Ising model. Look at a stochastic (or deterministic) transformation of the Gibbs measures, and identify the sets $n G(\gamma)$ of the parameter space (such as the space 
spanned by the temperature and field variables) for which the image measure is non-Gibbs as a lattice-measure, in the sense of the product topology. Define $n G(\mathrm{MF})$ as the parameter set for which the (corresponding) image measure of the (corresponding) mean-field model is non-Gibbs. Then, we expect the following, expressing Kac-compatibility of non-Gibbsianness.

Conjecture 1. "Usually"

$$
n G(\gamma) \rightarrow n G(M F)
$$

in the Kac-(long range)-limit $\gamma \downarrow 0$.

Such a statement is reminiscent of the stability of the phase-diagram at low temperatures (proved in the framework of Pirogov-Sinai theory), structural stability in bifurcation theory etc. A precise statement will depend on the transformation and on the precise definition of the models under consideration. In particular one may need sufficiently high lattice dimensions. To develop full proofs presumably will be non-trivial and at present the above conjecture is more a research program then a theory.

We will however describe in the examples below what the theorem means more precisely and which steps at this point are missing.

\subsubsection{Kac-Limit Background}

Let us start by reviewing what is the relation between translation-invariant Kac-models and mean-field models, see also 4. A general motivation for the introduction of Kac-models is the hope that by taking first the infinite-volume limit, and then the Kac-limit $\gamma \downarrow 0$, one obtains the corresponding mean-field model. Historically this was motivated first by the desire to justify the Maxwell construction (equal-area rule) from a microscopical model. The first fundamental result relating Kac and mean field and expressing a "Kac-compatibility" is the Lebowitz-Penrose theorem. ${ }^{(44)}$ It says that the free energy of the lattice-model converges to the convex (envelope of the) free energy of the corresponding meanfield model. The Lebowitz-Penrose theorem holds in considerable generality for various lattice spin models with short-range interactions, in any dimension.

However, as the example of ferromagnetic Kac-model in 1d shows, convergence to a model with flat mean-field rate-function (a free energy with a phase transition) does not need to be accompanied by non-uniqueness of the infinitevolume Gibbs measure on the lattice. Indeed, to understand the Gibbs measures is a much more subtle (and dimension-dependent) question. While uniqueness holds in one dimension for any finite $\gamma$, the Gibbs measure behaves in a non-trivial way and concentrates at a mesoscopic scale on profiles with jumps between values close to the corresponding (positive or negative) mean-field magnetisation. ${ }^{(8,9)}$ 
In lattice dimensions $d \geq 2$ however, Kac-compatibility is expected to hold for translation-invariant ferromagnetic models also on the level of the phase diagram, that is, the set of Gibbs measures. In the special case of the standard KacIsing model (10) this problem is indeed settled. The existence of ferromagnetically ordered low-temperature states in the Kac-model, and moreover the convergence of the critical inverse temperature $\beta_{c}(\gamma) \rightarrow \beta_{c}(\mathrm{MF})=1$ was proved independently in refs. 5 and 7 . Both proofs are based on spin-flip symmetry and don't generalize to models without symmetry between the phases, see however (6). We stress that the Kac-model might behave different from a nearest neighbour model. An example of this is the new result of ref. 25 on two-dimensional three-state Potts models. For a recent general description of Kac-limit results we refer to ref. 50 .

\subsubsection{Decimation of Standard Kac-Ising Model to a Sublattice}

As our first illustration we consider now the decimation transformation of the ferromagnetic Kac-Ising model to a sublattice $S$ that is kept fixed, independently of the Kac-parameter $\gamma$. Denote by $1-p$ the density of $S$ in $\mathbb{Z}^{d}$. Think of $d=2$ and the sub-lattice $S=(2 \mathbb{Z})^{2}$ for concreteness, so that $p=\frac{3}{4}$. Denote by $n G(\gamma)$ the range of critical inverse temperatures for which the projected measure is nonGibbsian. We will argue that

$$
n G(\gamma) \rightarrow\left[\frac{1}{p}, \infty\right)=n G(\mathrm{MF})
$$

as $\gamma \downarrow 0$. To analyze Gibbsianness of the transformed Gibbs measures before the Kac-limit, and analyze badness of a configuration $\eta$, we go through the standard program for lattice systems. This leads us to analyze the quenched model with Hamiltonian for the a spin-model on the "three-quarter lattice" $S^{c}=\mathbb{Z}^{d} \backslash S$ given by

$$
-\frac{1}{2} \sum_{i, j \in S^{c}} J_{\gamma}(i-j) \sigma_{i} \sigma_{j}-\sum_{i \in S^{c}}\left(\sum_{j \in S} J_{\gamma}(i-j) \eta_{j}\right) \sigma_{i}
$$

Consider a checkerboard-configuration $\eta=\eta_{\text {spec }}$. We claim that it is a bad configuration for low temperatures at sufficiently small $\gamma$. Indeed, the $\eta$-dependent term in the Hamiltonian is neutral and translation-invariant, so that we can expect phase-coexistence for the quenched model (13). Note that the site-dependent effective magnetic field $\sum_{j \in S} J_{\gamma}(i-j) \eta_{j}$ acting on the spin $\sigma_{i}$ becomes small uniformly in the Kac-limit, and so a mild modification of the contour-construction given in refs. 5 or 7 should prove that there is indeed phase coexistence at low temperature.

What about the critical inverse temperature $\beta_{c}\left(\gamma, S^{c}, \eta_{\text {spec }}\right)$ ? We see that the effective interaction-strength on the three-quarter lattice $S^{c}$ is reduced by the knocking out of $S$, in this way reducing the effective inverse temperature of the 
model to $p \beta$. Again, using (the modification of) 5,7 up to the critical point should prove indeed $\beta_{c}\left(\gamma, S^{c}, \eta_{\text {spec }}\right) \rightarrow \frac{1}{p}=\frac{4}{3}$. If we further assume that the "worst" bad configuration is indeed given by the checkerboard configuration, we have indeed proved the 1.h.s. of (12).

Finally consider the corresponding mean-field set-up at zero external field. Here a Curie-Weiss model of size $N$ is projected to a subset of size $(1-p) N$, and continuity of the single-site conditional probabilities of the projected measure at fixed magnetisation of the conditioning is investigated. A computation shows that the range of inverse temperatures for which non-Gibbsianness holds is indeed given by $\left[\frac{1}{p}, \infty\right)$ (see ref. 37). In this example, we do not need the disordered model as a tool for the mean-field analysis, in contrast to what happens on the finite-dimensional and Kac models. This is the case because conditioning on a finite fraction of the spins being fixed does not depend on the location of these spins; indeed in mean-field models talking about the location of spins on a lattice does not make sense.

Let us contrast this with a result from ref. 35. Here the following opposite result was proved: Start with a Kac-Ising model, with or without site-dependent magnetic fields. Take block-averages over blocks with width $l$ (sufficiently) smaller than the range $\frac{1}{\gamma}$ of the Kac-potential. Then the resulting measure is Gibbs.

\subsubsection{Decimation of Kac Models Without Symmetry}

Let us consider more generally Hamiltonians of the form

$$
H(\sigma)=\frac{1}{2} \sum_{i, j} \Phi_{i-j}^{\gamma}\left(\sigma_{i}, \sigma_{j}\right)+\sum_{i} U\left(\sigma_{i}\right)
$$

with $\sigma_{i}$ taking values in a finite set, the interaction kernel $\Phi_{i-j}^{\gamma}$ has finite range $1 / \gamma$, is "smooth enough" and $\sum_{k}\left\|\Phi_{k}^{\gamma}\right\|_{\infty}=O(1)$. No symmetry of the interaction under permutation of the phases is assumed.

Project a Gibbs-state of this model to a $\gamma$-independent sublattice $S$. What can we say about non-Gibbsianness? Do we expect convergence of the region of inverse temperatures and fields for which the decimated model is non-Gibbs to the corresponding mean-field values as $\gamma \downarrow 0$ ?

To start with, we review what is known about the translation-invariant phases. The work of refs. 6 and 53 proves the existence of ordered phases at lowtemperatures, uniformly in (sufficiently small) $\gamma$. It provides essential steps of a Kac-Pirogov-Sinai theory, and gives a good understanding of the structure of the phases. What is lacking however is the control up to the critical temperature of the corresponding mean-field model. So, Kac-compatibility is only partially proved already on the level of translation-invariant phases. 
In order to analyze badness of a configuration $\eta$ of the projected lattice we need to understand whether resp. for what values of parameters the quenched model

$$
\frac{1}{2} \sum_{i, j \in S^{c}} \Phi_{i-j}^{\gamma}\left(\sigma_{i}, \sigma_{j}\right)+\sum_{i \in S^{c}}\left(U\left(\sigma_{i}\right)+\sum_{j \in S} \Phi_{i-j}^{\gamma}\left(\sigma_{i}, \eta_{j}\right)\right)
$$

has a phase transition.

This should be possible when we reverse the order of choices, taking a periodic $\eta$ first, and looking for values of the parameters for which there is coexistence, using the method of refs. 6 and 53. A difficulty here is that the range of temperature for which one is able to prove coexistence will in general be non-uniform in the period. Such an argument would show the existence of bad configurations at a discrete $\gamma$-dependent set of parameter-values of the model. So, this method would provide a proof of non-Gibbsianness that is not exhaustive over the whole presumed region of non-Gibbsianness. To improve this and prove non-Gibbsianness for a continuum of parameter values, again randomizing to choose bad configurations would be necessary. To prove this rigorously one would need a fully developed nonsymmetric random Kac-Pirogov-Sinai-theory. In such a generality such a theory does not (yet) exist. For an outline of corresponding results in the random field Kac-Ising model (with symmetric random field distribution) see ref. 4. Finally, to study a.s. Gibbsianness such a theory would have to allow for non-independent Gibbs-quenching, too. All of this would be highly non-trivial. As in the short-range situation the steps involving randomized bad configurations are expected to work only in $d \geq 3$, whereas the arguments before should be valid in $d \geq 2$.

\subsubsection{Non-Gibbsian Quenched Measures in Mean Field}

As remarked before, the non-Gibbsianness of the quenched joint measures $K(n, \sigma)($ cf. $(6,9))$ has a mean-field analogue, as first shown in ref. 37. Physically, this again expresses the fact that an annealed description of such measures in terms of a Morita "grand potential" does not work. The analogy goes quite far, in that the set of bad configurations has measure zero or full measure in the mean-field situations according to the behaviour of the analogous model in finite dimensions (Zero measure but non-empty for dilution, full measure in the phase transition regime of the random field model).

Thus for these quenched measures the connection between disorder and nonGibbsianness again is "intrinsic", whereas disordered methods as a tool may or may not occur in the mean-field analysis of systems without disorder (in fact it occurs for the Glauber-evolved situation, ${ }^{(39)}$ but not for decimation ${ }^{(37)}$ ). 
Furthermore, for mean-field quenched models one can perform a rather complete analysis of the Morita approach and where and how it breaks down and what one still can learn from it (see refs. 32, 38 and references mentioned there).

Again, it would be of interest to see if one can connect the analyses of the mean-field and the lattice models via a Kac limit, although again we expect that some of the same complicated technical problems we mentioned before would need to be solved (in particular developing a general non-symmetric random Pirogov-Sinai theory).

\section{SUMMARY AND CONCLUSIONS}

We have reviewed two different connections which exist between the study of non-Gibbsian measures and of disordered systems. It turns out that there are two main types of connections:

1) Results and insights developed in the study of disordered systems often provide useful tools to study non-Gibbsianness.

2) Disordered systems give rise to non-Gibbsian measures.

We have discussed these in some detail, mentioning various examples and also the various physical meanings of these non-Gibbsian results, including in renormalization group theory, non-equilibrium questions and the Morita approach to disordered systems. Also we mentioned how these ideas are developed in a mean field setting, and we have suggested how a link may be developed in the Kac limit.

\section{ACKNOWLEDGEMENTS}

We thank all our colleagues with whom we have worked on or discussed these topics for all they have taught us, and Joel Lebowitz and Pierluigi Contucci for their invitation to contribute to this volume.

\section{REFERENCES}

1. L. Bertini, E. M. N. Cirillo and E. Olivieri, Gibbsian properties and convergence of the iterates for the block-averaging transformation. Mark. Proc. Rel. Fields 10:381-394 (2004).

2. L. Bertini, E. M. N. Cirillo and E. Olivieri, Graded cluster expansion for lattice systems. Comm. Math. Phys. 258:405-443 (2005).

3. L. Bertini, E. M. N. Cirillo and E. Olivieri, Renormalization group in the uniqueness region Weak Gibbsianity and convergence. Comm. Math. Phys. 261:323-378 (2006).

4. A. Bovier and C. Külske, Coarse-graining techniques for (random) Kac models, Interacting Stochastic Systems Springer Berlin. (2005), pp. 11-28.

5. A. Bovier and M. Zahradník, The low-temperature phase of Kac-Ising models. J. Stat. Phys. 87:311-332 (1997). 
6. A. Bovier and M. Zahradník, Cluster expansions and Pirogov-Sinai theory for long range spin systems. Markov Proc. Rel. Fields 8:443-478 (2002).

7. M. Cassandro, R. Marra and E. Presutti, Corrections to the critical temperature in $2 \mathrm{D}$ Ising systems with Kac potentials. J. Stat. Phys. 78:1131-1138 (1995).

8. M. Cassandro, E. Orlandi and P. Picco, Typical configurations for one-dimensional random field Kac model. Ann. Prob. 27:1414-1467 (1999).

9. M. Cassandro, E. Orlandi and E. Presutti, Interfaces and typical Gibbs configurations for onedimensional Kac potentials. Prob. Theor. Rel. Fields 96:57-96 (1993).

10. J. Bricmont and A. Kupiainen, Phase transition in the 3D random field Ising-model. Comm. Math. Phys. 116:539-572 (1988).

11. J. Bricmont, A. Kupiainen and R. Lefevere, Renormalisation group pathologies and the definition of Gibbs states. Comm. Math. Phys. 194:359-388 (1998).

12. J. Bricmont, A. Kupiainen and R. Lefevere, Renormalizing the renormalization group pathologies. Phys. Rep. 348:5-31 (2001).

13. T. W. Burkhardt and J. M. J. van Leeuwen, Progress and problems in real-space renormalization. In vol. 30, Topics in Current Physics, Real-Space renormalization, T. W. Burkhardt and J. M. J. van Leeuwen, (eds.) , pp. 1-31 Springer, Berlin (1982).

14. D. Dereudre and S. Roelly, Propagation of Gibbsianness for infinite-dimensional gradient Brownian diffusions. J. Stat. Phys. 121:511-551 (2005).

15. A. C. D. van Enter, R. Fernández and A. D. Sokal, Regularity properties of position-space renormalization-group transformations: Scope and limitations of Gibbsian theory. J. Stat. Phys. 72:879-1167 (1993).

16. A. C. D. van Enter, R. Fernández, F. den Hollander and F. Redig, Possible loss and recovery of gibbsianness during the stochastic evolution of gibbs measures. Comm. Math. Phys. 226:101-130 (2002).

17. A. C. D. van Enter and J. Lörinczi, Robustness of the non-Gibbsian property: Some examples. J. Phys. A, Math. Gen. 29:2465-2473 (1996).

18. A. C. D. van Enter, C. Külske and C. Maes, Comment on: Critical behavior of the randomly spin diluted 2D Ising model: A grand ensemble aproach, by R. Kühn. Phys. Rev. Lett. 84:6134 (2000).

19. A. C. D. van Enter, C. Maes, R. H. Schonmann and S. B. Shlosman, The Griffiths singularity random field. In R. A. Minlos, S. B. Shlosman and Yu. M. Suhov (eds.) On Dobrushin's Way: From Probability Theory to Statistical Physics pp. 51-58, AMS Translations: Series 2 (Providence, RI, USA, 2000)

20. A. C. D. van Enter, K. Netočný and H. G. Schaap, On the Ising model with random boundary condition. J. Stat. Phys. 118:997-1056 (2005).

21. A. C. D. van Enter and E. A. Verbitskiy, On the variational principle for Generalized Gibbs Measures. Mark. Proc. Rel. Fields 10:411-434 (2004).

22. R. Fernández, Gibbsianness and non-Gibbsianness in lattice random fields. In A. Bovier, J. Dalibard, F. Dunlop, A. C. D. van Enter and F. den Hollander, (eds.), Proceedings 2005 les Houches Summerschool on Mathematical Statistical Physics, to appear, (2006).

23. J. Fröhlich and J. Z. Imbrie, Improved perturbation expansion for disordered systems: Beating Griffiths singularities. Comm. Math. Phys. 96:145-180 (1984).

24. H.-O. Georgii, Gibbs Measures and Phase Transitions. (de Gruyter, Berlin 1988).

25. T. Gobron and I. Merola, Phase transition induced by increasing the range of interaction in Potts Model, mp-arc 05-272.

26. R. B. Griffiths, Nonanalytic behavior above critical point in a random ising ferromagnet, Phys. Rev. Lett. 23:17-19 (1969).

27. O. Häggström and C. Külske, Gibbs properties of the fuzzy Potts model on trees and in mean field. Markov Proc. Rel. Fields 10:477-506 (2004). 
28. R. B. Israel, Some generic results in mathematical physics. Mark. Proc. Rel. Fields 10:517-521 (2004).

29. M. Kac, G. Uhlenbeck and P. C. Hemmer, On the van der Waals theory of vapour-liquid equilibrium. I. Discussion of a one-dimensional model. J. Math. Phys. 4:216-228 (1963); II. Discussion of the distribution functions. J. Math. Phys. 4:229-247 (1963); III. Discussion of the critical region. J. Math. Phys. 5:60-74 (1964).

30. T. Kennedy, Majority Rule at Low Temperatures for the Square Lattice with $b=2$ and for the Triangular lattice. J. Stat. Phys. 86:1089-1107 (1997).

31. R. Kühn, Equilibrium ensemble approach to disordered systems. 1. General theory and exact results. Z. Phys. B 100:231-242 (1996).

32. R. Kühn, Gibbs versus Non-Gibbs in the equilibrium approach to disordered systems. Mark. Proc. Rel. Fields 10:523-545 (2004).

33. R. Kühn and G. Mazzeo, Critical behaviour of the 2-d spin diluted ising model via the equilibrium ensemble approach. Phys. Rev. E 60:3823-3836 (1999).

34. C. Külske, (Non-)Gibbsianness and phase transitions in random lattice spin models. Mark. Proc. Rel. Fields 5:357-383 (1999).

35. C. Külske, On the Gibbsian nature of the random field Kac model after block-averaging. J. Stat. Phys. 104:991-1012 (2001).

36. C. Külske, Weakly Gibbsian Representations for joint measures of quenched lattice spin models. Prob. Theor. Rel. Fields 119:1-30 (2001).

37. C. Külske, Analogues of non-Gibbsianness in joint measures of mean field models. J. Stat. Phys. 112:1101-1130 (2003).

38. C. Külske, How non-Gibbsianness helps a metastable Morita minimizer to provide a stable free energy. Mark. Proc. Rel. Fields 10:547-564 (2004).

39. C. Külske and A. Le Ny, Spin-flip dynamics of the Curie-Weiss model: Loss of Gibbsianness with possibly broken symmetry. Preprint, mp-arc 05-357, 2005.

40. C. Külske, A. Le Ny and F. Redig, Relative entropy and variational properties of generalized Gibbsian measures. Ann. Prob. 32:1691-1726 (2004).

41. C. Külske and F. Redig, Loss without recovery of Gibbsianness during diffusion of continuous spins. Prob. Theor. Rel. Fields 135(3): 428-456 (2006).

42. J. L. Lebowitz and C. Maes, The effect of an external field on an interface, entropic repulsion. $J$. Stat. Phys. 46:39-49 (1987).

43. J. L. Lebowitz and R. H. Schonmann, Pseudo-free energies and large deviations for non gibbsian FKG measures. Prob. Theor. Rel. Fields 77:49-64 (1988).

44. J. Lebowitz and O. Penrose, Rigorous treatment of the Van der Waals Maxwell theory of the liquid-vapour transition. J. Math. Phys. 7:98-113 (1966).

45. R. Lefevere and H. Tasaki, High-temperature expansion for nonequilibrium steady states in driven lattice gases. Phys. Rev. Lett. 94:200601 (2005).

46. C. Maes, A. van Moffaert and F. Redig. Almost Gibbsian versus weakly Gibbsian measures. Stoch. Proc. Appl. 79:1-15 (1999).

47. T. Morita, Statistical mechanics of quenched solid solutions with application to magneticallly dilute alloys. J. Math. Phys. 5:1402-1405 (1964).

48. Mark. Proc. Rel. Fields, 10 Proceedings Gibbs-non-Gibbs, Eindhoven. (2004).

49. C. M. Newman, Topics in Disordered Systems. Lecture in Mathematics, ETH-Zürich. Birkhäuser, Basel, (1997).

50. E. Presutti, Scaling Limits in Statistical Mechanics and Microstructures in Continuum Mechanics. Book in preparation, 2006.

51. R. H. Schonmann, Projections of Gibbs measures may be non-Gibbsian. Comm. Math. Phys. 124:1-7 (1989). 
52. M. Zahradník, On the structure of low-temperature phases in three-dimensional spin models with random impurities: A general Pirogov-Sinai approach. In R. Kotecký (ed.), Phase Transitions: Mathematics, Physics, Biology, Singapore, World Scientfic (1992), pp. 225-237.

53. M. Zahradník, Cluster expansions of small contours in abstract Pirogov-Sinai models. Markov Process. Related Fields 8:383-441 (2002). 